\title{
EXISTENCE OF GLOBAL SOLUTION OF A NONLINEAR WAVE EQUATION WITH SHORT-RANGE POTENTIAL
}

\author{
V. GEORGIEV and K. IANAKIEV \\ Section of Mathematical Physics, Institute of Mathematics, Bulgarian Academy of Sciences \\ Acad. G. Bonchev str. bl. 8, 1113 Sofia, Bulgaria
}

The existence of a global solution for the Cauchy problem for the nonlinear wave equation

$$
\left(\partial_{t t}-\Delta\right) u=F\left(u^{\prime}, u^{\prime \prime}\right), \quad t \geq 0, x \in \mathbb{R}^{3},
$$

is established by S. Klainerman [6] under a suitable algebraic condition called the null condition on the quadratic nonlinearity in $F\left(u^{\prime}, u^{\prime \prime}\right)$. The global or "almost global" solution of the corresponding mixed problem has been studied by Y. Shibata and Y. Tsutsumi [10], P. Datti [1] and P. Godin [2].

The main goal of this work is to study the nonlinear wave equation involving a linear perturbation of short-range type, i.e. we shall consider the Cauchy problem for the perturbed wave equation

$$
\left(\partial_{t t}-\Delta\right) u+q(x) x=F\left(x, u^{\prime}, u^{\prime \prime}\right),
$$

where $q(x)$ is a smooth potential, which is nonnegative and decays sufficiently rapidly at infinity. More precisely, we assume that

$$
q(x) \geq 0, \quad \partial_{r} q(x) \leq 0, \quad|q(x)| \leq C\left(1+|x|^{2}\right)^{-2},
$$

where $\partial_{r}$ is the radial derivative $\partial_{r}=\sum_{j=1}^{3}\left(x_{j} / r\right) \partial_{j}, r=|x|$.

Even in the simple case $q \equiv 0$, the results of F. John [5] show that the solution blows up for some choices of the nonlinearity $F$. That is why we assume that

$$
F=Q\left(x, u^{\prime}, u^{\prime \prime}\right)+C\left(u^{\prime}, u^{\prime \prime}\right),
$$

where $C(v, w)$ is a smooth function such that $C(v, w)=O\left((|v|+|w|)^{3}\right)$ near $(c, w)=(0,0)$, while $Q$ has the form

$$
Q\left(x, u^{\prime}, u^{\prime \prime}\right)=\sum_{1 \leq \alpha \leq 2} \sum_{1 \leq \beta \leq 2} q_{\alpha, \beta}(x) \partial^{\alpha} u \partial^{\beta} u,
$$




$$
\partial^{\alpha}=\partial_{t}^{\alpha_{0}} \partial_{1}^{\alpha_{1}} \partial_{2}^{\alpha_{2}} \partial_{3}^{\alpha_{3}}, \partial_{0}=\partial_{t}, \partial_{j}=\partial_{x_{j}}, j=1,2,3 .
$$

The quadratic perturbation in (4) is assumed to satisfy the estimate

$$
\left|q_{\alpha, \beta}(x)\right| \leq C(1+|x|)^{-1} .
$$

Under the assumptions described above we consider the Cauchy problem for the nonlinear wave equation (2) with initial data

$$
u(0, x)=\varepsilon u_{0}(x), \quad \partial_{t} u(0, x)=\varepsilon u_{1}(x),
$$

where $u_{0}, u_{1} \in C_{0}^{\infty}\left(\mathbb{R}^{3}\right)$.

Our main result is the following.

THEOREM. Under the assumptions $\left(\mathrm{H}_{1}\right),\left(\mathrm{H}_{2}\right)$, there exists a sufficiently small positive number $\varepsilon_{0}$ such that for $0<\varepsilon \leq \varepsilon_{0}$ the Cauchy problem (2), (5) has a unique global solution.

The main idea of the proof of a similar result for compact linear perturbations and mixed boundary problems for (1) is due to Y. Shibata and T. Tsutsumi and it is based on the application of the local energy decay for the corresponding linear perturbations of the classical wave equation. The presence of a non-compact perturbation in (2) causes a similar approach to meet essential difficulties. For this reason we follow another idea proposed by S. Klainerman, namely, we use a suitable modification of the usual conformal killing vector field

$$
K_{0} u=\left(1+t^{2}+|x|^{2}\right) \partial_{t} u+2 t r \partial_{r} u+2 t u .
$$

More precisely, we consider the vector field

$$
K=K_{0}+t \xi\left(\partial_{t}+\partial_{r}\right)+t \xi / r
$$

where $\xi=\xi(r)$ is a suitable radial function satisfying

$$
0 \leq r \xi^{\prime}(r) \leq \xi(r), \quad \xi^{\prime \prime}(r) \leq 0, \quad \xi(0)=0 .
$$

The function $\xi(r)$ serves to compensate the influence of the potential $q$ so that one can control the $L^{2}$ norm of the solution as well as the $L^{2}$ norms of $L^{\alpha} Z^{\beta} u$, where $L_{j}=t \partial_{j}+x_{j} \partial_{t}, j=1,2,3$, and

$$
\begin{aligned}
\left\{Z_{1}, \ldots, Z_{8}\right\}= & \left\{\partial_{\mu}, \mu=0,1,2,3 ;\right. \\
& \left.\Omega_{k l}=x_{k} \partial_{l}-x_{l} \partial_{k}, 1 \leq k<l \leq 3 ; S=t \partial_{t}+\sum_{j=1}^{3} x_{j} \partial_{j}\right\} .
\end{aligned}
$$

This modification of the conformal vector field allows one to derive a precise $L^{2}-L^{2}$ conformal estimate of the perturbed linear wave equation

$$
(\square+q) u+\gamma^{\lambda \mu} \partial_{\lambda} \partial_{\mu} u=F
$$

Namely, we have the following 
Proposition 1. Suppose the properties (6) are fulfilled and the assumption $\left(\mathrm{H}_{1}\right)$ holds. Let $\gamma^{\lambda \mu}(t, x), 0 \leq \lambda, \mu \leq 3$, satisfy the inequalities

$$
(1+|x|)(1+t+|x|) \sum_{|\beta| \leq 1}\left|L^{\beta} \gamma^{\lambda \mu}(t, x)\right| \leq \delta
$$

for $t \geq 0, x \in \mathbb{R}^{3}$ and some $\delta \in(0,1)$. Then the solution $u(t, x)$ of (8) satisfies the estimate

$$
\begin{aligned}
\sum_{|\alpha|+|\beta| \leq 1}\left\|L^{\alpha} Z^{\beta} u(t, \cdot)\right\|_{L^{2}}^{2} \leq C \sum_{|\alpha|+|\beta| \leq 1}\left\|L^{\alpha} Z^{\beta} u(0, \cdot)\right\|_{L^{2}}^{2} \\
\quad+C \int_{0}^{t} \int_{\mathbb{R}^{n}} F(s, x) K u(s, x) d x d s \\
+C \delta \int_{0}^{t}(1+s)^{-1} \sum_{|\alpha|+|\beta| \leq 1}\left\|L^{\alpha} Z^{\beta} u(s, \cdot)\right\|_{L^{2}}^{2} d s \\
\quad+C \int_{0}^{t}(1+s)^{-1}\left\|\nabla_{t, x} u(s, \cdot)\right\|_{L^{2}} \sum_{|\alpha|+|\beta| \leq 1}\left\|L^{\alpha} Z^{\beta} u(s, \cdot)\right\|_{L^{2}} d s
\end{aligned}
$$

with some constant $C>0$.

To obtain an estimate for higher order derivatives of the solution we use only the generators (7) of the conformal group in $\mathbb{R}^{1+3}$. The main advantage of this choice of generators is that the assumption $\left(\mathrm{H}_{1}\right)$ implies $\left|Z^{\alpha} q(x)\right| \leq C\left(1+|x|^{2}\right)^{-2}$ for any multiindex $\alpha$. This basic property enables one to derive a higher order decay estimate. We avoid the use of the boosts $L_{j}, j=1,2,3$, because of the decay of the commutator of $L_{j}$ and the potential. Even application of the generators (7) is not sufficient to estimate the $L^{2}$ norms of the higher order derivatives of the solution. To estimate the $L^{2}$ derivatives of order $N$ we consider the modified scaling

$$
S^{\#}=S+\xi \partial_{r}+\xi / r
$$

where $\xi$ is some function. To control the sup-norm of the solution we need an $L^{\infty}-L^{2}$ estimate for the linear wave equation involving only the generators chosen above in (7). To do this we apply the approach proposed in [9] and based on the estimation of the resolution operator for the linear wave equation (8) with $\gamma^{\lambda \mu}=0$.

Let $N \geq 1$ be a fixed integer. We introduce the various $L^{2}$ norms

$$
e_{N}(t, u)=\sum_{\substack{|\alpha|+|\beta| \leq N+1 \\|\alpha| \leq 1}}\left\|L^{\alpha} Z^{\beta} u(t, \cdot)\right\|_{L^{2}},
$$




$$
\begin{aligned}
f_{N}(t, u) & =\sum_{\substack{|\alpha|+|\beta| \leq N \\
|\alpha| \leq 1,|\gamma|=1}}\left\|L^{\alpha} \partial^{\gamma} Z^{\beta} u(t, \cdot)\right\|_{L^{2}}+\left\|L^{\alpha} \Omega^{\gamma} Z^{\beta} u(t, \cdot)\right\|_{L^{2}}, \\
g_{N}(t, u) & =\sum_{|\beta| \leq N,|\gamma|=1}\left\|\partial^{\gamma} Z^{\beta} u(t, \cdot)\right\|_{L^{2}},
\end{aligned}
$$

as well as some weighted $L^{\infty}$ norms

$$
\left\{\begin{array}{l}
e_{n, \text { int }}(t, u)=\sup _{0 \leq \tau \leq t} \sum_{|\beta| \leq n}\left\|\tau_{+}^{2} Z^{\beta} u(\tau, x)\right\|_{L^{\infty}(|x| \leq h \tau)}, \\
e_{n, \operatorname{ext}}(t, u)=\sup _{0 \leq \tau \leq t} \sum_{|\beta| \leq n}\left\|\tau_{+} \tau_{-} Z^{\beta} u(\tau, x)\right\|_{L^{\infty}(|x| \geq h \tau)}
\end{array}\right.
$$

where $n=N / 2+1, \tau_{ \pm}=1+|\tau \pm| x||$ and $0<h<1$ is suitably chosen. These norms play an important role in our investigation. Assuming the $L^{\infty}$ norms $e_{n, \text { ext }}(t, u)$ and $e_{n, \text { int }}(t, u)$ to be bounded, say

$$
e_{n, \text { ext }}(t, u)+e_{n, \text { int }}(t, u) \leq 1,
$$

we prove that the $L^{2}$ norms (10) satisfy the estimate

$$
(1+t)^{-\sigma} e_{N}(t, u)+f_{N}(t, u) \leq \eta(\varepsilon) .
$$

Here $\sigma$ is a positive constant independent of $\varepsilon$, while $\eta(\varepsilon)$ tends to 0 as $\varepsilon$ tends to 0 . The proof of this estimate is based on Proposition 1.

From the representation of the solution one can follow the approach developed by $\mathrm{H}$. Pecher under the algebraic conditions $\left(\mathrm{H}_{1}\right)$ imposed on the potential $q$. This allows one to derive a suitable $L^{\infty}-L^{\infty}$ estimate for the linear wave equation (8) with $\gamma^{\lambda \mu}=0$.

Proposition 2. Let $u(t, x)$ be a solution of (2) with initial data (5). There exists $\delta>0$ such that for any sufficiently small $\varepsilon$ the estimate

$$
e_{n, \text { ext }}(t, u)+e_{n, \text { int }}(t, u) \leq \delta
$$

implies

$$
e_{n, \mathrm{ext}}(t, u)+e_{n, \text { int }}(t, u) \leq \eta(\varepsilon),
$$

where $\eta(\varepsilon) \rightarrow 0$ as $\varepsilon \rightarrow 0$.

Proof of Theorem. The above estimates show that for any $T>0$ the sup-norm

$$
\sup _{0 \leq t<T} \sup _{x \in \mathbb{R}^{3}} \sum_{|\alpha| \leq 2}\left|\partial_{t, x}^{\alpha} u(t, x)\right|
$$

is bounded by some constant $C>0$, independent of $T$. The continuation principle completes the proof of the Theorem.

\section{References}

[1] P. Datti, Long time existence of classical solutions to a non-linear wave equation in exterior domains, Ph.D. Dissertation, New York University, 1985. 
[2] P. Godin, Long time behaviour of solutions to some nonlinear rotation invariant mixed problems, Comm. Partial Differential Equations 14 (3) (1989), 299-374.

[3] L. Hörmander, The Analysis of Linear Partial Differential Operators, Vol. I, Distribution Theory and Fourier Analysis, Springer, New York 1983.

[4] - Non-linear Hyperbolic Differential Equations, Lectures 1986-1987, Lund 1988.

[5] F. John, Blow-up for quasi-linear wave equations in three space dimensions, Comm. Pure Appl. Math. 34 (1981), 20-51.

[6] S. Klainerman, The null condition and global existence to nonlinear wave equations, in: Lectures in Appl. Math. 23, Part 1, Amer. Math. Soc., Providence, R.I., 1986, 293-326.

[7] - Uniform decay estimates and the Lorentz invariance of the classical wave equation, Comm. Pure Appl. Math. 38 (1985), 321-332.

[8] C. Morawetz, Energy decay for star-shaped obstacles, Appendix 3 in: P. Lax and R. Phillips, Scattering Theory, Academic Press, New York 1967.

[9] H. Pecher, Scattering for semilinear wave equations with small initial data in three space dimensions, Math. Z. 198 (1988), 277-288.

[10] Y. Shibata and Y. Tsutsumi, On global existence theorem of small amplitude solutions for nonlinear wave equations in exterior domains, ibid. 191 (1986), 165-199. 